\title{
Analysis on the Incidence, Staging and Treatment of Carcinoma Cervix at Delta Medical College and Hospital of Bangladesh
}

\author{
Parvin A Banu1, Naheed Rukhsana ${ }^{2}$, Jebunnessa Yasmin³, Lutfun Nahar4, \\ Sadiqur R Malik 5
}

\begin{abstract}
Background: Cervical cancer remains the most common cancer among women in this part of the world. Bangladesh has the highest level of incidence and mortality rates due to cervical cancer among women. Cervical cancer is a preventable disease by screening and treatment of pre-invasive condition. Unfortunately, there is no effective screening program in Bangladesh. Objective: The objective was to analyze the clinico-pathologic characteristics and, subsequently the therapy delivered to the patients. Materials and method: This cross sectional study was done from January to December 2011 in oncology division of Delta Medical College and Hospital, Dhaka, Bangladesh. During this period a total of 2264 female carcinoma patients were registered. Out of them 523 patients were with the diagnosis of carcinoma of cervix. Evaluation and characterization of patients with carcinoma cervix were done according to the age group, clinical stages and surgical status. External beam radiotherapy (EBRT) and doses, brachytherapy doses and fractions, time interval between EBRT and brachytherapy, neo-adjuvant and concurrent chemotherapy status were the factors taken into consideration for the analysis of treatment of these patients. Results: The study revealed that the incidence of carcinoma cervix was $23 \%$ and highest incidence was between 40-50 years of age group, most of the patients were in Stage IIB (34\%) and IIIB (28\%) and 31\% with unknown stage. About 44\% patients were referred postoperatively and most of them with unknown stage. Almost $90 \%$ patients received EBRT, 24\% patients received concurrent chemo-radiation, $46 \%$ of them completed 5 cycles of concurrent chemotherapy and $8.6 \%$ patients received neo-adjuvant chemotherapy. About $69 \%$ patients received brachytherapy with HDR Co-60 sources and $23 \%$ of patients received their brachytherapy treatment after 12 weeks of completion of EBRT. Conclusion: Lack of proper clinical evaluation and documentation, delay in referral and lack of implementation of a standard protocol for the treatment of carcinoma cervix are the major obstacles in our country.
\end{abstract}

Keywords: Carcinoma cervix; incidence; treatment.

1. Professor (C.C.), Oncology Division, Delta Medical College and Hospital, Dhaka, Bangladesh.

2. Assistant Professor, Oncology Division, Delta Medical College and Hospital, Dhaka, Bangladesh.

3. Assistant Professor, Oncology Division, Delta Medical College and Hospital, Dhaka, Bangladesh.

4. Assistant Professor, Oncology Division, Delta Medical College and Hospital, Dhaka, Bangladesh .

5. Chief Radiation Oncology Physicist, Oncology Division, Delta Medical College and Hospital, Dhaka, Bangladesh.

Correspondence: Prof. Dr. Parvin A Banu. e-mail:pabanu@yahoo.com

\section{Introduction}

According to the global WHO statistics, 7.9 million deaths occur each year due to cancer. Of these deaths, around $70 \%$, i.e. 5.5 million are now occurring in the developing world. Cancer of the cervix is the second most common cancer in women in the world, with an estimated 529,409 new cases and 274,883 deaths in 2008. ${ }^{1}$ Bangladesh has the highest level of incidence and mortality rates due to cervical cancer among women. The prevalence of cervical cancer in women of Bangladesh is around 25-30\%/100,000. ${ }^{2}$ 
Bangladesh has a population of 50.19 million women aged 15 years and older who are at risk of developing cervical cancer. Current estimates indicate that every year in Bangladesh 17,686 women are diagnosed with cervical cancer and 10,364 die from the disease. Cervical cancer ranks as the second most frequent cancer in women of Bangladesh and the second most frequent cancer among women between 15 and 44 years of age. Data is not yet available on the human papilloma virus (HPV) burden in the general population of Bangladesh. However, in Southern Asia, the region Bangladesh belongs to, $\sim 7.9 \%$ of women in the general population is estimated to harbor cervical HPV infection at a given time. ${ }^{2}$

HPV infection is one of the most common sexually transmitted diseases. Roughly half of all cervical cancers worldwide contain the onco-gene HPV16; other important high risk types are HPV18, 45 and 31.3 One study showed that the prevalence of HPV in sex workers of Bangladesh was $75.8 \%$ whereas for the high risk group it was $49.8 \%$. The most prevalent high risk HPV types, in order of prevalence rate, were HPV16, HPV18, HPV58, HPV45, HPV31 and HPV33. Both HPV16 and HPV18 were present in 21\% of the cases. 4

Cervical cancer is a preventable disease by screening and treatment of pre-invasive condition. Unfortunately, there is no effective screening program in Bangladesh. Risk factors for cervical cancer include early age at first intercourse, history of multiple sexual partners, and smoking. It is more common in low socio-economic classes. . $^{-7}$

A study done in Mumbai, India showed that nearly $70 \%$ of cervical cancer patients present at Stages III and IV of the disease. Around $20 \%$ of women who develop cervical cancer die within the first year of diagnosis and the 5 year relative survival rate is $50 \%$. The majority of cases are squamous cell carcinoma and adenocarcinoma is less common. ${ }^{8}$

\section{Materials and method}

This cross sectional study was carried out at oncology division of Delta Medical College and Hospital during the period of January to December 2011 to analyze the incidence, staging and treatment modalities of cancer cervix. Total 2264 female cancer patients were registered during the study period. Among them 523 (23\%) patients with age ranges from 20-80 years were diagnosed as carcinoma of uterine cervix. All patients underwent gynecological examination in this centre and extent of the disease for each patient was documented, staging was done (where possible) according to FIGO staging. Routine and relevant investigations were performed.

All the patients except those who failed to continue the treatment were treated with external beam radiotherapy (EBRT) to pelvis either in this centre or in outside hospital. In this centre it was done either by LINAC or Co-60 machine. Prescribed EBRT dose was 46 to 50 Gy in 25 fractions over 5 weeks. Few patients who had pelvic lymph node metastases on CT scan were treated with extended field radiation to the para-aortic nodes also. Indicated patients received HDR brachytherapy with Co-60 BEBIG Multisource unit, installed first time in Bangladesh. 9 They received brachytherapy either with tandem and ovoids, vaginal cylinder, tandem and vaginal cylinder or interstitial application.
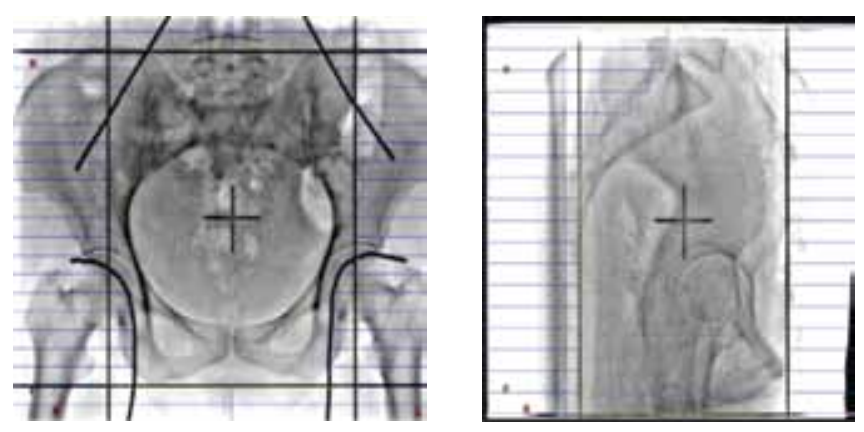

Fig 1: Simulation X-rays of EBRT to Pelvis(AP \& Lateral)

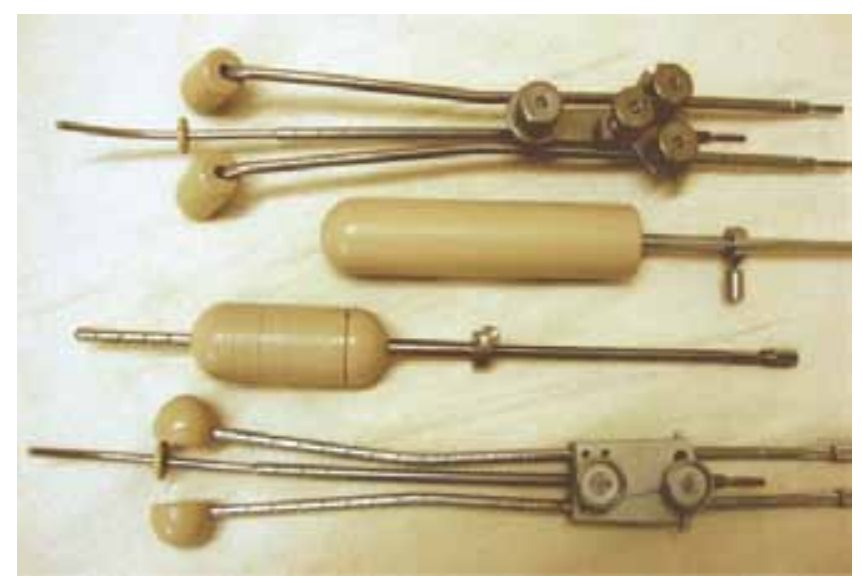

Fig 2: Brachytherapy applicators:

Fletcher suite, Manchester and vaginal cylinder 

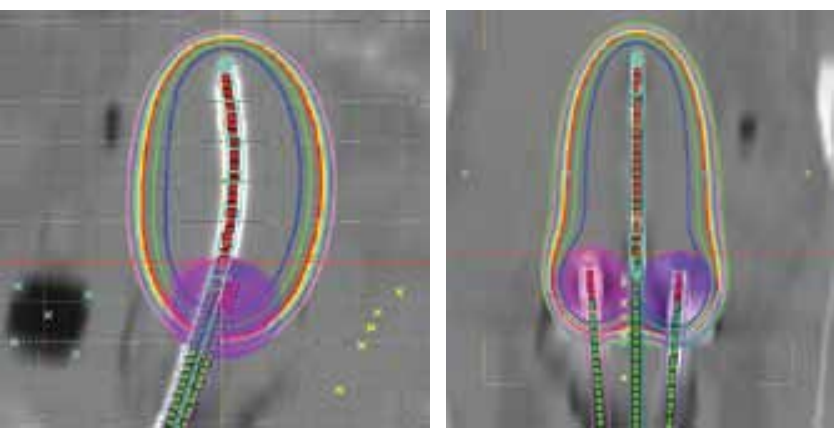

Fig 3: Pear-Shaped isodose distribution of intra-cavitary application of cervical cancer

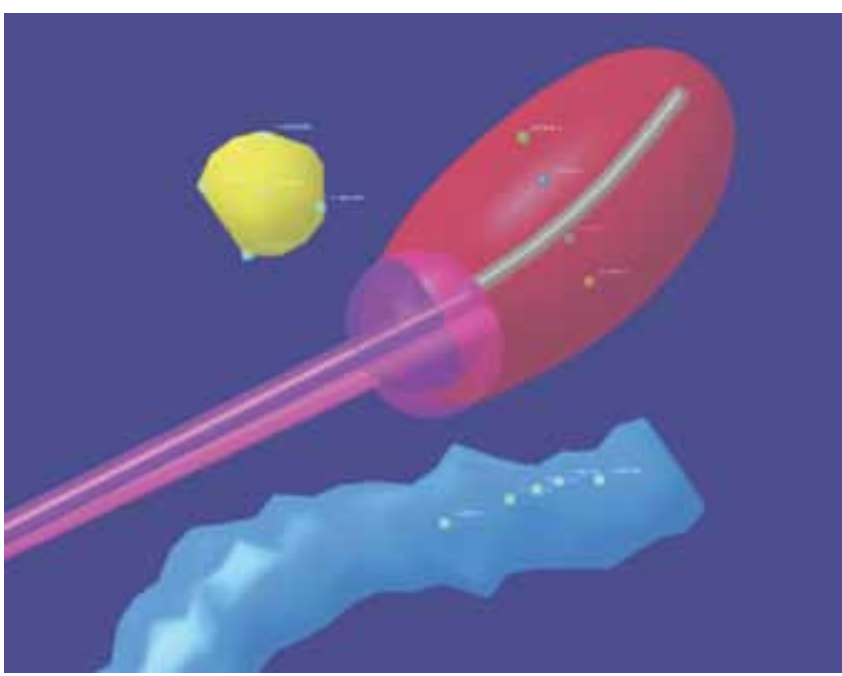

Fig 4: 3D Volume delineation for brachytherapy treatment
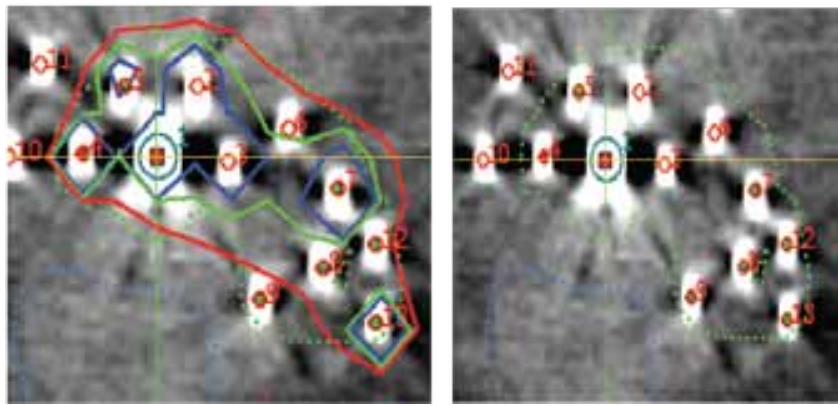

Fig 5: Interistial application of parametrial recurrence of a cervical cancer

Patients with advanced stage of carcinoma cervix were treated with chemotherapy according to FNCA (Forum for Nuclear Co-operation in Asia) Cervix-IV protocol (Fig 6). 10 Concurrent chemotherapy was given with Inj. Cisplatin $40 \mathrm{mg} / \mathrm{m}^{2} \mathrm{I} / \mathrm{V}$ weekly scheduled for 6 cycles.

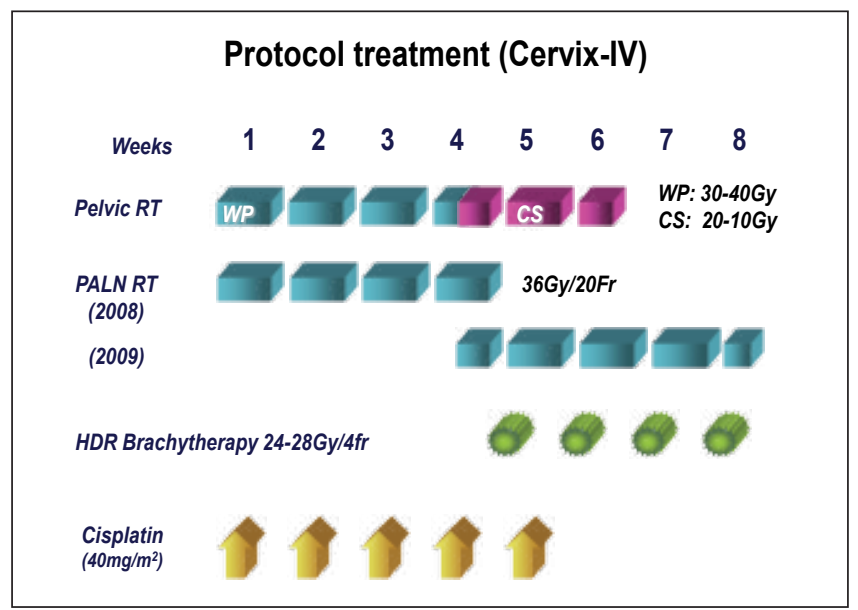

\section{CCRT + Prophylactic PALN-RT}

for Stage IIB-IIIB Cervical Cancer with PLN (+) Aim to decrease distant meta (incl. PALN metastases)

Patientaccrual, 2008.

Fig 6: FNCA Cervix-IV Protocol for the treatment of Advanced Stage Carcinoma Cervix

\section{Results}

Among 2264 female cancer patients total 523 (23\%) patients were diagnosed as having carcinoma cervix. Fig 7 shows the incidence of carcinoma cervix.

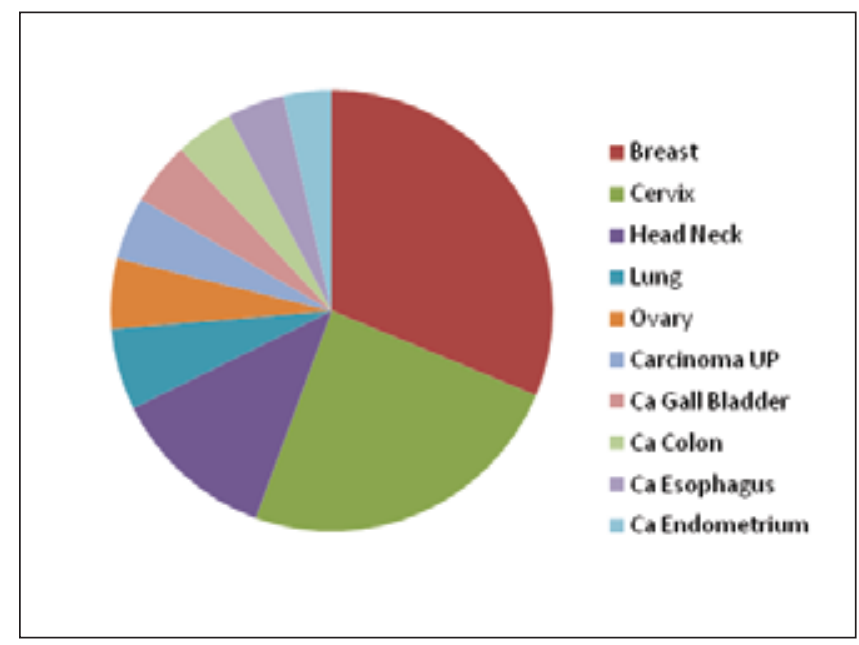

Fig 7: Distribution of female cancer patients

Regarding the age incidence majority (39.38\%) of the patients were within 41-50 years of age group (Table I). 
Table I: Frequency of carcinoma cervix in different age groups $(\mathrm{N}=\mathbf{5 2 3})$

\begin{tabular}{ccc}
\hline Age groups (years) & Frequency & Percentage \\
\hline $20-30$ & 22 & 4.2 \\
$31-40$ & 113 & 21.6 \\
$41-50$ & 206 & 39.3 \\
$51-60$ & 123 & 23.5 \\
$61-70$ & 48 & 9.1 \\
$71-80$ & 11 & 2.3 \\
\hline
\end{tabular}

Table II shows the histopathological subtype of carcinoma cervix. Histopathologically most of the patients $(93 \%)$ were diagnosed as squamous cell carcinoma, followed by adenocarcinoma (5.7\%) and adeno-squamous cell carcinoma (1.1\%).

Table II: Histopathological type of carcinoma cervix $(N=523)$

\begin{tabular}{lcc}
\hline Subtypes & Number of patients & Proportion \\
\hline Squamous cell carcinoma & 487 & $93 \%$ \\
Adenocarcinoma & 30 & $5.7 \%$ \\
Adeno-squamous cell carcinoma & 6 & $1.1 \%$ \\
\hline
\end{tabular}

Among the patients 233 (44.55\%) were referred to this centre postoperatively. Out of these referred cases 40 patients $(17.18 \%)$ came with recurrence of disease at vault of vagina years after surgery. The time interval between surgery and development of recurrence was from 1-6 years.

Table III shows that staging of the carcinoma cervix was documented in $361(69.02 \%)$ patients. The highest proportion was at Stage IIB (34.07\%) followed by Stage IIIB (27.70\%). Staging could not be explored in remaining $162(30.97 \%)$ patients.

Table III: Different stages of carcinoma cervix $(n=361)$

\begin{tabular}{ccc}
\hline Stages & Number of patients & Proportion \\
\hline IA & 3 & $0.83 \%$ \\
IB & 23 & $6.37 \%$ \\
IIA & 62 & $17.17 \%$ \\
IIB & 123 & $34.07 \%$ \\
IIIA & 11 & $3.04 \%$ \\
IIIB & 100 & $27.70 \%$ \\
IVA & 32 & $8.86 \%$ \\
IVB & 7 & $1.93 \%$ \\
\hline
\end{tabular}

Almost all (90.43\%) the patients received EBRT to pelvis. One hundred ninety one (40.38\%) patients received EBRT at Delta Medical College and Hospital and $282(59.61 \%)$ patients received EBRT in outside hospitals. Remaining 50 patients $(9.5 \%)$ did not turn up for any kind of treatment after initial registration. Among the 191 patients receiving EBRT at Delta Medical College and Hospital 90\% patients received EBRT to pelvis by LINAC and $10 \%$ by Co- 60 machine. Almost all patients completed their treatment within 35-45 days with few exceptions who took up to 50 days to complete EBRT. More than $90 \%$ patients tolerated external radiotherapy without any serious acute toxicity. Only some hematological and non-hematological toxicities were seen in patients who received concurrent chemo-radiation.

Out of 523 patients 359 (68.64\%) patients received HDR brachytherapy. Most of the patients who received EBRT at this centre received their brachytherapy treatment within 4 weeks of completion of EBRT but the patients who were referred from outside hospital for brachytherapy, most of them received their treatment beyond 4 weeks (Table IV).

Table IV: Time interval in receiving brachytherapy in weeks $(n=359)$

\begin{tabular}{ccc}
\hline Weeks & Number of Patients & Percentage (\%) \\
\hline 1 & 47 & 13.09 \\
2 & 38 & 10.58 \\
3 & 38 & 10.58 \\
4 & 64 & 17.82 \\
5 & 9 & 2.50 \\
6 & 10 & 2.78 \\
7 & 10 & 2.78 \\
8 & 26 & 7.24 \\
9 & 8 & 2.22 \\
10 & 6 & 1.67 \\
11 & 4 & 1.11 \\
12 & 15 & 4.17 \\
$>12$ & 84 & 23.39 \\
\hline
\end{tabular}


Out of these 359 patients, 152 received brachytherapy with tandem and ovoids; 199 patients (all postoperative cases) received treatment with vaginal cylinder, 6 patients with tandem and vaginal cylinder and 2 patients with interstitial application.

According to our institutional protocol locally advanced stages i.e. Stage IIB, IIIA, IIIB and IVA cases were treated with 7 Gy weekly in 3 fractions and few cases with 6 Gy weekly in 4 fractions using BEBIG Multisource HDR with Co-60 for postoperative patients who were in early stages of cancer. ${ }^{9}$ They received 5 Gy in 2 fractions to the vault and upper third of the vagina each one week apart. High risk patients like histological high grade, cut margin involvement, Stage IIA received 6-7 Gy in 2-3 fractions.

Rectal and bladder doses were calculated according to ICRU-38 guidelines and were within permissible limits. Acute and late toxicities were within tolerable limits.

Out of 523 patients, 128 (24.47\%) patients received concurrent chemotherapy with Inj. Cisplatin $40 \mathrm{mg} / \mathrm{m}^{2}$ $\mathrm{I} / \mathrm{V}$ weekly. Information regarding concurrent chemotherapy was not always available for patients which were referred from outside hospitals. Forty five patients $(8.6 \%)$ referred from outside received neo-adjuvant chemotherapy prior to referral to this centre. Most of the patients (46\%) could complete up to 5 cycles of chemotherapy and only $3 \%$ patients could complete the full 6 cycle schedule (Table V). Many patients could not receive concurrent chemotherapy due to hematological and non-hematological toxicities or refused to continue chemotherapy.

Table V: Cycles of concurrent chemo and radiotherapy $(\mathbf{n}=128)$

\begin{tabular}{ccc}
\hline Number of cycles & Number of patients & Proportion \\
\hline 1 & 8 & $6.25 \%$ \\
2 & 7 & $5.46 \%$ \\
3 & 16 & $12.5 \%$ \\
4 & 34 & $26.56 \%$ \\
5 & 59 & $46.09 \%$ \\
6 & 4 & $3.12 \%$ \\
\hline
\end{tabular}

\section{Discussion}

This study revealed that the incidence of carcinoma cervix is $23 \%$ which almost represents the national data of Bangladesh. ${ }^{2}$ Approximately $70-75 \%$ of cervical carcinomas are of squamous cell type and we also found a similar pattern. ${ }^{11}$ Most of the carcinoma cervix patients were diagnosed at advanced stage and similar ovservation was made by Dinshaw et al. 8

It is advisable to start brachytherapy treatment within two weeks of completion of EBRT to get the maximum biological response, so patients should be referred for brachytherapy immediately after completion of EBRT. Concurrent chemotherapy should be given in advanced stages of carcinoma cervix as randomized clinical trials showed improved survival with concurrent chemo-radiation. ${ }^{12,13}$ But this study explored that a major proportion of patients were sent for brachytherapy after a long delay after initial EBRT even after 7-8 months. It is the combined outcome of ignorance and scarcity of brachytherapy facility. It is to be mentioned that this specialized centre is equipped with both EBRT and brachytherapy facilities and also expertise for providing such sophisticated radiotherapies along with chemotherapy whenever needed. The study also revealed that a good proportion of patients were referred after surgery without providing documents of preoperative clinical staging. Some patients also came with recurrence which might be due to ignorance and lack of health awareness among the common people as well as health care providers or delay in referral.

In Bangladesh, cancer cervix is one of the leading cancers and cause of death in women 2 ; hence emphasis should be given on screening and early diagnosis. A national consensus for the treatment guidelines for carcinoma cervix is necessary. Proper gynecological examination and staging must be done before decision of treatment. Inadequate surgery like sub-total hysterectomy prevents the patients to receive curative treatment, especially brachytherapy, which is a very important part of the treatment of cervical cancer. It should be emphasized that treatment of cancer cervix must be a team work by gynaecologists and oncologists. So strict protocol should be followed for carcinoma cervix patients starting from initial evaluation and treatment and necessary referral thereafter. 


\section{References}

1. World Health Organization: Cancer in developing countries: facing the challenge [Internet]. [cited 2012 March 3]. Available from: http://www.who.int/dg/speeches/2010/iaea_forum 20100921/en/ index.html.

2. WHO/ICO Information Centre on HPV and Cervical Cancer (HPV Information Centre). Human papillomavirus and related cancers in Bangladesh. Summary report 2010 [Internet]. [cited 2012 March 3]. Available from: http://www.who.int/hpvcentre.

3. Begum J, Hossain MA. Pap test for screening of carcinoma of cervix. Teachers Association Journal. $2006 ; 19(2): 76-80$

4. Sultana S, Huq M, Alam A, Mitra DK, Gomes DJ. Prevalence and genotyping of human papillomavirus in female with high - risk behavior in Dhaka, Bangladesh. Bangladesh J Microbiol. 2008;25(1):65-68

5. Christopherson WM, Parker JE. Relation of Cervical cancer to early marriage and childbearing. $\mathrm{N}$ England $\mathrm{J}$ Med. 1965;273: 235-39.

6. Winkelstein WJ. Smoking and cervical cancer-current status: a review. Am J Epidemiology. 1990;131:945-57.

7. Rotkin ID. Epidemiology of cancer of the cervix. 3. Sexual characteristic of a cervical cancer population. AM J Public Health Nations Health. 1967;57(5):815-29.

8. Dinshaw K, Mishra G, Shastri S, Badwe R, Kerkar R, Ramani S, et al. Determinants of compliance in a cluster randomized controlled trial on screening of breast and cervix cancer in Mumbai, India. I. Compliance to screening. Oncology. 2007;73(3-4):145-53.
9. Malik SR, Banu PA, Rukhsana N. A comprehensive study on HDR brachytherapy treatments of cervical cancers: using the first Co-60 BEBIG Multisource Unit in Bangladesh. J Contemp Brachyther. 2011;3(2):96-105.

10. FNCA Cervix-IV (Ongoing Phase II Trial): Concurrent chemo-radiotherapy to the pelvis and prophylactic para-aortic radiotherapy for locally advanced cervical cancer with enlarged pelvic lymph node [Internet]. [cited 2012 March 3]. Available from: http://www.fnca.mext.go.jp/english/mu/e_ws_2012.ht $\mathrm{ml}$.

11. Holschneider $\mathrm{CH}$. Premalignant \& malignant disorders of the uterine cervix. In: Decherney AH, Nathan L, Goodwin TM, Laufer N, editors. Current diagnosis \& treatment: Obstetrics \& Gynecology. 10th ed. New York: McGraw-Hill; 2007. p. 833-54.

12. Rose PG, Bundy BN. Chemo radiation for locally advanced cervical cancer. Journal of Clinical Oncology. 2002;20(4):891-93.

13. Kato S, Ohno T, Thephamongkhol K, Chansilpa Y, Yuxing Y, Devi CRB, et al. Multi-institutional phase II clinical study of concurrent chemoradiotherapy for locally advanced cervical cancer in East and Southeast Asia. International Journal of Rad. Oncology Biology Physics. 2010;77(3):751-57. 